\title{
Large Deep White Matter Lesions May Predict Futile Recanalization in Endovascular Therapy for Acute Ischemic Stroke
}

\author{
Yohei Tateishi $^{a, c} \quad$ Dolora Wisco $^{c}$ Junya Aoki ${ }^{b}$ Pravin George ${ }^{c}$ \\ Irene Katzan $^{c}$ Gabor Toth ${ }^{c}$ FerdinandHui ${ }^{c}$ Muhammad S. Hussain ${ }^{c}$ \\ Ken Uchino ${ }^{c}$ \\ ${ }^{a}$ Cerebrovascular Center, Department of Neurology and Strokology, Nagasaki University \\ Hospital, Nagasaki City, and bepartment of Neurological Science, Graduate School of \\ Medicine, Nippon Medical School, Tokyo, Japan; ${ }^{~}$ Cerebrovascular Center, Cleveland Clinic, \\ Cleveland, Ohio, USA
}

\section{Key Words}

Acute stroke · Endovascular therapy · Magnetic resonance imaging · Deep white matter ·

Outcome

\begin{abstract}
Objective: This study investigated whether large ischemic lesions in the deep white matter (DWM) on pretreatment diffusion-weighted MRI (DWI) predict futile recanalization. Methods: Consecutive acute stroke patients with anterior circulation ischemia who underwent successful arterial recanalization with thrombolysis in cerebral infarction grade $2 \mathrm{~b}$ or 3 were enrolled. A large DWI-DWM lesion was defined as a hyperintense lesion in the DWM on initial DWI, located mainly between the anterior and posterior horns of the lateral ventricle. The Alberta Stroke Program Early CT score on CT and DWI and stroke volume on initial DWI were recorded. Stroke severity was assessed using the National Institutes of Health Stroke Scale (NIHSS) score. Futile recanalization was defined as a 30-day modified Rankin scale score of 3-6 despite successful recanalization. Univariate and multivariate regression analyses were performed to identify predictors of futile recanalization. Results: In 35 of 46 patients (76\%) with successful recanalization, futile recanalization was observed in 20 patients (57\%). Patients with futile recanalization were older (median age 74 vs. 58 years; $p=0.053$ ), had higher initial NIHSS scores (median 17 vs. 9; $\mathrm{p}=0.042$ ), and a higher prevalence of large DWI-DWM lesions (45 vs. $9 \% ; p=0.022$ ). Logistic regression analysis showed that a large DWI-DWM lesion was an independent predictor of futile recanalization (OR 13.97; 95\% CI 1.32-147.73; $p=0.028$ ). Conclusion: Patients with large preintervention DWI-DWM lesions may be poor candidates for endovascular therapy.


Tateishi et al.: Large Deep White Matter Lesions May Predict Futile Recanalization in Endovascular Therapy for Acute Ischemic Stroke

\section{Introduction}

Early recanalization of occluded cerebral arteries is usually associated with good outcomes in patients with acute ischemic stroke who undergo intravenous or intra-arterial thrombolysis or mechanical thrombectomy $[1,2]$. Recent controlled clinical trials of acute endovascular therapy that selected patients based on the time from onset of stroke symptoms failed to demonstrate that endovascular therapy was better than intravenous thrombolysis with recombinant tissue plasminogen activator (IV rt-PA) [3, 4]. Failure of endovascular therapy in both clinical trials and clinical practice may be partly due to the subset of stroke patients who have poor outcomes despite successful recanalization. In previous reports about mechanical thrombectomy, only one third to half of all ischemic stroke patients with partial or complete recanalization had good outcomes, with modified Rankin scale scores of $0-2$ [5-10]. A review by Hussein et al. [11] found that the rate of futile recanalization in acute ischemic stroke patients who underwent endovascular recanalization was $49 \%$.

Diffusion-weighted MRI (DWI) can detect irreversibly injured brain tissue after acute ischemic stroke, and it is therefore useful in patients who are candidates for acute recanalization therapy. The initial DWI lesion volume, Alberta Stroke Program Early CT score (ASPECTS) on DWI, and location of hyperintense lesions on DWI were reported to be good predictors of outcome in patients who underwent endovascular therapy [12,13]. Patients with basal ganglia infarction on DWI had more disability at discharge, a longer hospital stay, and a higher rate of hemorrhagic infarction after endovascular therapy [14]. A large basal ganglia infarction may imply poor collateral flow with early infarction because the deep white matter (DWM) is an internal arterial border zone. We hypothesized that a large ischemic lesion in the DWM on DWI might predict poor outcomes in patients with successful recanalization after endovascular therapy.

\section{Subjects and Methods}

Patients

Using our acute stroke endovascular database, acute stroke patients with anterior circulation ischemia who underwent endovascular therapy within $8 \mathrm{~h}$ of onset between June 2010 and May 2011 were retrospectively identified. All patients with partial or complete recanalization [thrombolysis in cerebral infarction (TICI) grade $2 \mathrm{~b}$ or 3 ] after endovascular therapy were included in this study. All patients underwent both CT and MRI, while perfusion-weighted MRI was performed in a subset of patients. Patients with contraindications for MRI, such as an implanted cardiac pacemaker or intracranial artery clipping, were excluded. The study was approved by the Institutional Review Board at the Cleveland Clinic (Cleveland, Ohio, USA).

\section{Patient Characteristics}

The following patient characteristics were recorded: age, sex, previous ischemic heart disease and ischemic stroke, and vascular risk factors (hypertension, diabetes mellitus, hyperlipidemia, and smoking). Vascular risk factors were defined as follows. Hypertension was defined as use of antihypertensive agents, a systolic blood pressure of $>140 \mathrm{~mm} \mathrm{Hg}$, or a diastolic blood pressure of $>90 \mathrm{~mm} \mathrm{Hg}$. Diabetes mellitus was defined as use of oral hypoglycemic agents or insulin, or a glycosylated hemoglobin level of $>6.5 \%$. Hyperlipidemia was defined as use of antihyperlipidemic agents or a serum cholesterol level of $>220 \mathrm{mg} / \mathrm{dl}$. Smoking was defined as having smoked cigarettes within the last 5 years. Stroke severity was assessed using the National Institutes of Health Stroke Scale (NIHSS) score. Patients who had received IV rt-PA prior to endovascular therapy were included in this study.

Acute Imaging

All patients underwent CT and MRI on admission. CT was performed before MRI unless imaging examinations were performed before arrival at our hospital. Standard noncontrast CT was performed using a multislice CT scanner (Sensation 64/16; Siemens, Munich, Germany) with 1.2-mm collimation and 4.5-mm 
Tateishi et al.: Large Deep White Matter Lesions May Predict Futile Recanalization in Endovascular Therapy for Acute Ischemic Stroke

slice thickness (S64), or 1.5-mm collimation and 4.8-mm slice thickness (S16). The patients were scanned from the skull base to the vertex with contiguous axial slices parallel to the orbitomeatal baseline. Noncontrast CT was followed immediately on the same scanner by CT angiography using a helical scan technique. The images were acquired after a single bolus intravenous injection of $80 \mathrm{ml}$ nonionic contrast agent (Iopromide; Bayer Schering Pharma AG, Berlin, Germany) into an antecubital vein at $4 \mathrm{ml} / \mathrm{s}$. The patients were scanned from the foramen magnum to the centrum semiovale or vertex with spiral $0.6-\mathrm{mm}$ collimation, 0.75-mm slice thickness, and 0.7-mm interval (S64), or spiral 0.75-mm collimation, 0.75-mm slice thickness, and $0.7-\mathrm{mm}$ interval (S16). MRI was performed using a commercially available echo planar instrument on a 1.5-tesla Magnetom Aera system with Syngo MR D11 software (Siemens). The neuroimaging protocol for acute stroke at our hospital includes DWI [repetition time (TR)/echo time (TE) 3,500/114 ms; b values 0 and $1,400 \mathrm{~s} / \mathrm{mm}^{2}$; field of view $230 \mathrm{~mm}$; slice thickness $5.0 \mathrm{~mm}$; base resolution 128; bandwidth 1,260 Hz], fluidattenuated inversion recovery pulse sequences (TR/TE 8,360/94 ms; inversion time 2,200 ms; flip angle $180^{\circ}$; field of view $210 \mathrm{~mm}$; slice thickness $4.0 \mathrm{~mm}$; base resolution 256; bandwidth $208 \mathrm{~Hz}$ ), dynamic susceptibility contrast perfusion-weighted MRI sequences (TR/TE 2,000/45 ms; flip angle $90^{\circ}$; field of view 230 $\mathrm{mm}$; slice thickness $5.0 \mathrm{~mm}$; base resolution 128; bandwidth 1,260 Hz).

\section{Treatment}

Patients who presented to a regional hospital or our hospital within $4.5 \mathrm{~h}$ of symptom onset were treated with IV rt-PA if there were no contraindications. The dose of IV rt-PA was $0.9 \mathrm{mg} / \mathrm{kg}$ according to the National Institute of Neurological Disorders and Stroke guidelines $[15,16]$.

If both an initial NIHSS score $\geq 8$ and concomitant large-vessel occlusion (cervical internal carotid artery, intracranial internal carotid artery, M1, and M2) on CT angiography were present, the endovascular team was activated. On the basis of these MRI results, all patients who had either clinical-DWI mismatch (clinical deficit out of proportion to DWI lesion, approximately $<70 \mathrm{ml}$ volume core infarct by visual inspection) or DWI-perfusion-weighted MRI mismatch (by visual inspection of the perfusion map) were immediately transported to the neuroradiography suite. The patients received either sedation (first-line) or general anesthesia with endotracheal intubation at the discretion of the neurointerventionalist and anesthesiologist. Groin access was obtained, and the target vessel was immediately catheterized for diagnostic angiography to confirm vessel occlusion. Mechanical thrombectomy was performed using a MERCI device (Concentric Medical Inc., Fremont, Calif., USA) or the Penumbra Aspiration System (Penumbra Inc., Alemeda, Calif., USA), and/or chemical lysis was performed using intra-arterial t-PA or glycoprotein IIb/IIIa inhibitors. Recanalization was assessed using the TICI criteria $[17,18]$. TICI 0 indicates no perfusion, TICI 1 indicates penetrating, but no distal branch filling, TICI 2a indicates perfusion with incomplete $(<50 \%)$ distal branch filling, TICI $2 \mathrm{~b}$ indicates perfusion with incomplete $(>50 \%)$ distal branch filling, and TICI 3 indicates full perfusion with filling of all distal branches. At the conclusion of the procedure, the patients were transported to the neurological intensive care unit for postprocedure care and monitoring. Standard stroke care and processes were implemented.

\section{Assessment of the Images}

All images were assessed by 2 experienced vascular neurologists (Y.T. or J.A.) who were blinded to the clinical information. Perfusion-weighted MRI reconstruction was performed using commercial software on a workstation supplied by the vendor (Siemens). The arterial input function was derived from the M1 segment of the middle cerebral artery or a branch in the hemisphere contralateral to the largest diffusion abnormality [19]. A mean-transit-time (MTT) map was used to detect the ischemic penumbra. A large DWI-DWM lesion was defined as a longitudinal hyperintense lesion in $>80 \%$ of the DWM that was located between the anterior and posterior horns of the lateral ventricle. The lateral expansion of a diffusion abnormality at the level of the corona radiata was not taken into account when the lesion was considered to be a large DWI-DWM lesion. The lesion was not considered to be a large DWI-DWM lesion if there was a portion of the DWM at the corona radiata (fig. 1). The ASPECTS was calculated on CT and DWI. Ischemic lesion volumes on DWI and MTT were calculated by multiplying the area of abnormal intensity outlined manually by slice thickness. The abnormal lesions on CT, DWI and MTT were visually defined by comparison with the contralateral nonaffected hemisphere. The window level and window width were chosen to obtain the best contrast between the lesion and the surrounding normal tissues. The occlusion site was diagnosed based on CT angiography or MR angiography. Considerable hemorrhagic transformation was defined as a parenchymal hematoma type 2 (a dense hematoma in $>30 \%$ of the infarcted area with a substantial space-occupying effect), or any hemorrhagic lesion outside the infarcted area [20]. The cerebral angiograms were 
Fig. 1. a A large ischemic lesion in the DWM on DWI. b, c DWI-DWM lesions that are not large.

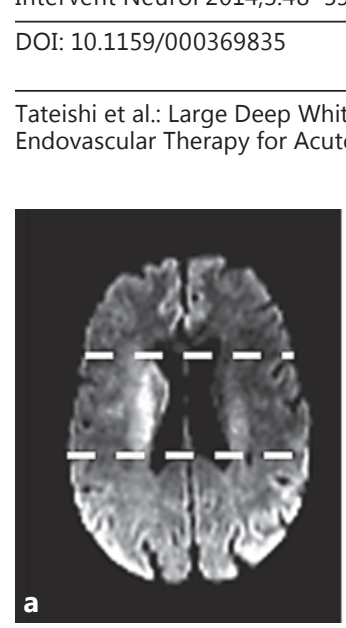

Tateishi et al.: Large Deep White Matter Lesions May Predict Futile Recanalization in Endovascular Therapy for Acute Ischemic Stroke
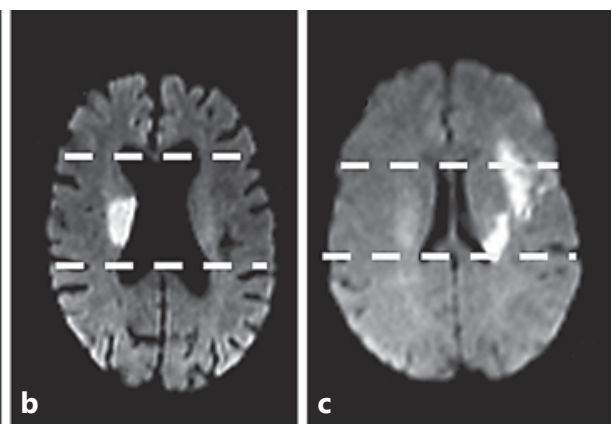

reviewed by experienced endovascular surgical neuroradiologists (M.S.H., G.T. or F.H.), and the TICI grades were recorded.

Analysis

Futile recanalization was defined as a 30-day modified Rankin scale score of 3-6 despite successful recanalization (TICI grade $2 \mathrm{~b}$ or 3 ). Clinical and imaging baseline parameters were compared between patients with futile and nonfutile recanalization using the Mann-Whitney $U$ test for numerical variables and Fisher's exact test for categorical variables. The data are presented as medians [interquartile range (IQR)] or frequencies (\%). To identify independent predictors of futile recanalization the time of presentation, multivariate logistic regression analysis was performed including all parameters with a p value $<0.1$ in the comparisons between groups. For continuous variables, the cutoff value was determined using the area under the receiver operating characteristic curve. Results were considered significant with $\mathrm{p}<0.05$. All analyses were performed using IBM SPSS software for Windows, version 18 (IBM, Armonk, N.Y., USA).

\section{Results}

Forty-six patients underwent endovascular therapy between June 2010 and May 2011. Thirty-five of the 46 patients (76\%), including 18 males (50\%), with a median age of 70 years (IQR 57-78 years) and a median NIHSS score of 13 (IQR 9-20), achieved successful recanalization (TICI grade $2 \mathrm{~b}$ or 3 ). Twenty patients (57\%) underwent follow-up MRI within $48 \mathrm{~h}$ of the recanalization therapy. They were all observed with patency of the treated artery. The period from the last time the patient was seen well to MRI was 278 min (IQR 224-391 min) and that to endovascular therapy was $374 \mathrm{~min}$ (IQR 295-464 min). Twenty-four of the 35 patients $(69 \%)$ underwent perfusion-weighted MRI. The occlusion site was the internal carotid artery in 15 patients (43\%) and the middle cerebral artery in 20 patients $(57 \%)$. Futile recanalization was identified in 20 of these 35 patients (57\%).

The results of the univariate analyses are shown in table 1 . Patients with futile recanalization were older (median age 74 vs. 58 years; $p=0.053$ ), had higher initial NIHSS scores (median 17 vs. 9; $\mathrm{p}=0.042$ ), had more frequently a large pretreatment DWI-DWM lesion ( 45 vs. 9\%; $\mathrm{p}=0.022$ ), and had a larger final ischemic lesion volume (median $37 \mathrm{vs.} 9 \mathrm{ml} ; \mathrm{p}=$ 0.003). Pretreatment ASPECTS on CT (median 9 vs. 9; $\mathrm{p}=0.527$ ) and on DWI (median 8 vs. 8; $\mathrm{p}=0.943$ ), initial ischemic lesion volume on DWI (median 21 vs. $14 \mathrm{ml} ; \mathrm{p}=0.230$ ), and MTT on MRI perfusion-weighted imaging (median 189 vs. $192 \mathrm{ml}$; $=0.976$ ) were similar between patients with and those without futile recanalization. There were no significant differences between the 2 groups in the times from onset to imaging or from onset to endovascular therapy. IV rt-PA before endovascular therapy was not associated with outcome.

Three variables with $\mathrm{p}<0.1$ on univariate analyses were identified. The areas under the receiver operating characteristic curves yielded cutoff values predicting futile recanalization 
Tateishi et al.: Large Deep White Matter Lesions May Predict Futile Recanalization in Endovascular Therapy for Acute Ischemic Stroke

Table 1. Comparisons between the 2 groups (futile vs. nonfutile recanalization)

\begin{tabular}{|c|c|c|c|}
\hline & $\begin{array}{l}\text { Futile recanalization } \\
(n=20)\end{array}$ & $\begin{array}{l}\text { Nonfutile recanalization } \\
(\mathrm{n}=15)\end{array}$ & $\mathrm{p}$ value \\
\hline Age, years & $74(65-78)$ & $58(53-77)$ & $0.053^{*}$ \\
\hline Male/female & $8 / 12$ & $10 / 5$ & $0.176^{\dagger}$ \\
\hline \multicolumn{4}{|l|}{ Past medical history } \\
\hline Ischemic stroke & $5(25)$ & $2(13)$ & $0.672^{\dagger}$ \\
\hline Ischemic heart disease & $8(40)$ & $2(13)$ & $0.134^{\dagger}$ \\
\hline \multicolumn{4}{|l|}{ Vascular risk factors } \\
\hline Hypertension & $16(80)$ & $8(53)$ & $0.144^{\dagger}$ \\
\hline Diabetes mellitus & $7(35)$ & $3(20)$ & $0.458^{\dagger}$ \\
\hline Dyslipidemia & $9(45)$ & $6(40)$ & $1.000^{\dagger}$ \\
\hline Smoking & $3(15)$ & $2(13)$ & $1.000^{\dagger}$ \\
\hline Atrial fibrillation & $5(25)$ & $5(33)$ & $0.712^{\dagger}$ \\
\hline Initial NIHSS score & $17(11-20)$ & $9(7-17)$ & $0.042^{*}$ \\
\hline \multicolumn{4}{|l|}{ Time, min } \\
\hline Last normal to MRI & $264(220-359)$ & $330(248-442)$ & $0.243^{*}$ \\
\hline Last normal to endovascular therapy & $334(292-462)$ & $395(331-495)$ & $0.382^{*}$ \\
\hline Found to MRI & $224(185-324)$ & $270(248-373)$ & $0.194^{*}$ \\
\hline Found to endovascular therapy & $306(240-454)$ & $364(314-459)$ & $0.113^{*}$ \\
\hline IV rt-PA prior to endovascular therapy & $11(55)$ & $8(53)$ & $1.000^{\dagger}$ \\
\hline \multicolumn{4}{|l|}{ Occlusion site } \\
\hline Internal carotid artery & $9(45)$ & $6(40)$ & $1.000^{\dagger}$ \\
\hline Middle cerebral artery & $11(55)$ & $9(60)$ & \\
\hline \multicolumn{4}{|l|}{ ASPECTS } \\
\hline CT & $9(8-9)$ & $9(8-10)$ & $0.527^{*}$ \\
\hline DWI & $8(6-9)$ & $8(6-8)$ & $0.943^{*}$ \\
\hline \multicolumn{4}{|l|}{ Ischemic lesion volume, $\mathrm{ml}$} \\
\hline DWI & $21(13-35)$ & $14(11-23)$ & $0.230^{*}$ \\
\hline MTT & $189(156-210)$ & $192(107-252)$ & $0.976^{*}$ \\
\hline Large DWI-DWM lesion & $9(45)$ & $1(7)$ & $0.022^{\dagger}$ \\
\hline TICI grade 3 recanalization & $7(35)$ & $3(20)$ & $0.458^{\dagger}$ \\
\hline Parenchymal hematoma type 2 incidence & $3(15)$ & 0 & $0.251^{\dagger}$ \\
\hline Final ischemic lesion volume, ml & $37(17-124)$ & $9(3-27)$ & $0.003^{*}$ \\
\hline
\end{tabular}

Data are median values with IQR in parentheses or numbers with percentages in parentheses. * MannWhitney U test. ${ }^{\dagger}$ Fisher's exact test.

Table 2. Predictors of futile recanalization: multivariate logistic regression analysis

\begin{tabular}{llrl}
\hline & p value & OR & $95 \%$ CI \\
\hline Large DWI-DWM lesion & 0.028 & 13.97 & $1.32-147.73$ \\
Initial NIHSS score $>15$ & 0.082 & 4.42 & $0.83-23.61$ \\
Age $>70$ years & 0.151 & 3.34 & $0.65-17.31$ \\
\hline
\end{tabular}

with high sensitivity and specificity as follows: age $>70$ years (sensitivity 65\%; specificity $69 \%$ ) and initial NIHSS score $\geq 15$ (sensitivity 65\%; specificity 75\%). Multivariate logistic regression analysis demonstrated that a large DWI-DWM lesion was an independent predictor of futile recanalization (OR 13.97; 95\% CI 1.32-147.73; $\mathrm{p}=0.028$; table 2). The sensitivity, specificity, positive predictive value, and negative predictive value of a large DWI-DWM lesion for predicting futile recanalization were $45,93,90$, and $56 \%$, respectively. 


\section{Discussion}

A large DWI-DWM lesion was the only independent predictor of unfavorable outcome at 30 days in patients with successful recanalization after endovascular therapy. The positive predictive value of a large DWI-DWM lesion for predicting futile recanalization was $90 \%$.

Some previous studies reported that a DWM lesion in acute ischemic stroke was related to poor outcome [21-24]. Loh et al. [14] reported that a diffusion abnormality throughout the basal ganglia and white matter in the territory of the middle cerebral artery was associated with worse disability at discharge, a longer hospital stay, and a higher rate of hemorrhage in acute ischemic stroke patients who underwent endovascular therapy, compared with other diffusion patterns. The results of the present study are consistent with those findings. Tracking analysis revealed that the area of the DWM was crossed by the corticospinal tract and many other cortico-subcortical and long-range intrahemispheric association fiber tracts [25]. The DWM is supplied by perforating branches of the middle cerebral artery and cortical branches of the anterior and posterior cerebral arteries [26, 27]. A large DWM lesion may therefore indicate that extensive infarction has already started because of poor collateral circulation. The state of the collateral circulation may be one of the strongest predictors of clinical outcome in patients with acute stroke $[28,29]$.

Older age and a higher initial NIHSS score were identified as predictors of futile recanalization in the present study. Hussein et al. [11] observed futile recanalization in $49 \%$ of patients who underwent endovascular therapy for acute ischemic stroke, and they found that age $>70$ years (OR 4.4; 95\% CI 1.9-10.5; p < 0.001) and an initial NIHSS score of 10-19 (OR $3.8 ; 95 \%$ CI 1.7-8.4; $\mathrm{p}<0.001$ ) were independent predictors of futile recanalization. Although the present multivariate analysis did not identify age $>70$ years or initial NIHSS score $\geq 15$ as independent predictors of futile recanalization, the results regarding age and initial NIHSS score were similar. An analysis of the PROACT II study that examined the efficacy of intra-arterial prourokinase for the treatment of acute stroke patients within $6 \mathrm{~h}$ of onset found that age $\leq 68$ years and NIHSS score $<20$ were associated with good outcome (modified Rankin scale score 0-2) after 90 days [30]. Among 3,864 patients who underwent endovascular clot retrieval, age $\geq 65$ years was associated with higher rates of in-hospital mortality and discharge to a long-term care facility than age $<65$ years $(29.7$ vs. $17.1 \%$; $p<0.0001$, and 54.1 vs. $47.6 \%$; $p<0.0001$, respectively) [31].

The results of the present study did not show an association between time to endovascular therapy and futile recanalization. Favorable outcomes were associated with early treatment in a pooled analysis of intravenous thrombolysis trials and with early recanalization in prospective endovascular therapy studies [32-34]. The lack of association between time to treatment and outcome in this study may be due to the small sample size or patient selection based on MRI findings. A post-hoc analysis of the DEFUSE2 study data showed that the ORs for a favorable response to treatment in patients with reperfusion versus those without reperfusion were not significantly different between patients who received early and late treatment, if they had target mismatch on MRI.

At this stage, we cannot assert that patients with large DWI-DWM lesions are not poor candidates for endovascular therapy. A randomized trial can determine whether this lesion pattern predicts unfavorable results from endovascular therapy. However, these results can be used to inform family discussions about whether endovascular therapy should be performed, since this pattern may be a poor prognostic indicator despite successful recanalization. To improve patient outcomes, a randomized trial to prove the usefulness of the pretreatment MRI lesion pattern is needed. 
Tateishi et al.: Large Deep White Matter Lesions May Predict Futile Recanalization in Endovascular Therapy for Acute Ischemic Stroke

This study was limited by its retrospective nature and the small number of patients. Patient selection based on CT and MRI findings before endovascular therapy limited the analysis to patients with relatively small infarcts and high ASPECTS.

\section{Conclusions}

A large DWI-DWM lesion before endovascular therapy predicts poor outcome in patients with successful recanalization. Patients with large DWI-DWM lesions may be poor candidates for endovascular therapy.

\section{Disclosure Statement}

The authors declare no conflicts of interest.

\section{References}

$\rightarrow 1$ Molina CA, Alexandrov AV, Demchuk AM, Saqqur M, Uchino K, Alvarez-Sabin J: Improving the predictive accuracy of recanalization on stroke outcome in patients treated with tissue plasminogen activator. Stroke 2004;35:151-156.

2 Rha JH, Saver JL: The impact of recanalization on ischemic stroke outcome: a meta-analysis. Stroke 2007;38: 967-973.

-3 Broderick JP, Palesch YY, Demchuk AM, et al: Endovascular therapy after intravenous t-PA versus t-PA alone for stroke. N Engl J Med 2013;368:893-903.

-4 Ciccone A, Valvassori L, Nichelatti M, Sgoifo A, Ponzio M, Sterzi R, Boccardi E; SYNTHESIS Expansion Investigators: Endovascular treatment for acute ischemic stroke. N Engl J Med 2013;368:904-913.

5 IMS II Trial Investigators: The Interventional Management of Stroke (IMS) II Study. Stroke 2007;38:21272135.

6 Furlan A, Higashida R, Wechsler L, et al: Intra-arterial prourokinase for acute ischemic stroke. The PROACT II study: a randomized controlled trial. Prolyse in Acute Cerebral Thromboembolism. JAMA 1999;282:20032011.

7 Alexandrov AV, Molina CA, Grotta JC, et al: Ultrasound-enhanced systemic thrombolysis for acute ischemic stroke. N Engl J Med 2004;351:2170-2178.

-8 Smith WS, Sung G, Starkman S, et al: Safety and efficacy of mechanical embolectomy in acute ischemic stroke: results of the MERCI trial. Stroke 2005;36:1432-1438.

-9 Smith WS: Safety of mechanical thrombectomy and intravenous tissue plasminogen activator in acute ischemic stroke. Results of the multi Mechanical Embolus Removal in Cerebral Ischemia (MERCI) trial, part I. AJNR Am J Neuroradiol 2006;27:1177-1182.

10 Smith WS, Sung G, Saver J, et al: Mechanical thrombectomy for acute ischemic stroke: final results of the Multi MERCI trial. Stroke 2008;39:1205-1212.

11 Hussein HM, Georgiadis AL, Vazquez G, Miley JT, Memon MZ, Mohammad YM, Christoforidis GA, Tariq N, Qureshi AI: Occurrence and predictors of futile recanalization following endovascular treatment among patients with acute ischemic stroke: a multicenter study. AJNR Am J Neuroradiol 2010;31:454-458.

-12 Yoo AJ, Verduzco LA, Schaefer PW, Hirsch JA, Rabinov JD, Gonzalez RG: MRI-based selection for intra-arterial stroke therapy: value of pretreatment diffusion-weighted imaging lesion volume in selecting patients with acute stroke who will benefit from early recanalization. Stroke 2009;40:2046-2054.

13 Costalat V, Lobotesis K, Machi P, et al: Prognostic factors related to clinical outcome following thrombectomy in ischemic stroke (RECOST Study). 50 patients prospective study. Eur J Radiol 2012;81:4075-4082.

14 Loh Y, Towfighi A, Liebeskind DS, et al: Basal ganglionic infarction before mechanical thrombectomy predicts poor outcome. Stroke 2009;40:3315-3320.

15 Adams HP Jr, Brott TG, Furlan AJ, et al: Guidelines for Thrombolytic Therapy for Acute Stroke: a Supplement to the Guidelines for the Management of Patients with Acute Ischemic Stroke. A statement for healthcare professionals from a Special Writing Group of the Stroke Council, American Heart Association. Stroke 1996; 27:1711-1718.

16 Practice advisory: thrombolytic therapy for acute ischemic stroke - summary statement. Report of the Quality Standards Subcommittee of the American Academy of Neurology. Neurology 1996;47:835-839.

17 Zaidat 00, Yoo AJ, Khatri P, et al: Recommendations on angiographic revascularization grading standards for acute ischemic stroke: a consensus statement. Stroke 2013;44:2650-2663. 
18 Higashida RT, Furlan AJ, Roberts $\mathrm{H}$, et al: Trial design and reporting standards for intra-arterial cerebral thrombolysis for acute ischemic stroke. Stroke 2003;34:e109-e137.

19 Thijs VN, Somford DM, Bammer R, Robberecht W, Moseley ME, Albers GW: Influence of arterial input function on hypoperfusion volumes measured with perfusion-weighted imaging. Stroke 2004;35:94-98.

20 Reeves MJ, Arora S, Broderick JP, et al: Acute stroke care in the US: results from 4 pilot prototypes of the Paul Coverdell National Acute Stroke Registry. Stroke 2005;36:1232-1240.

-21 Stoeckel MC, Wittsack HJ, Meisel S, Seitz RJ: Pattern of cortex and white matter involvement in severe middle cerebral artery ischemia. J Neuroimaging 2007;17:131-140.

22 DeVetten G, Coutts SB, Hill MD, Goyal M, Eesa M, O’Brien B, Demchuck AM, Kirton A; MONITOR and VISION study groups: Acute corticospinal tract Wallerian degeneration is associated with stroke outcome. Stroke 2010;41:751-756.

23 Seitz RJ: Stroke recovery: the pyramid in focus. Neurology 2010;74:276-277.

24 Kawano H, Hirano T, Inatomi Y, Terasaki T, Yonehara T, Uchino M: Presence of deep white matter lesions on diffusion-weighted imaging is a negative predictor of early dramatic improvement after intravenous tissue plasminogen activator thrombolysis. Cerebrovasc Dis 2010;30:230-236.

-25 Rosso C, Colliot O, Valabrègue R, Crozier S, Dormont D, Lehéricy S, Samson Y: Tissue at risk in the deep middle cerebral artery territory is critical to stroke outcome. Neuroradiology 2011;53:763-771.

-26 Mayer PL, Kier EL: The controversy of the periventricular white matter circulation: a review of the anatomic literature. AJNR Am J Neuroradiol 1991;12:223-228.

27 Nonaka H, Akima M, Hatori T, Nagayama T, Zhang Z, Ihara F: Microvasculature of the human cerebral white matter: arteries of the deep white matter. Neuropathology 2003;23:111-118.

28 Christoforidis GA, Mohammad Y, Kehagias D, Avutu B, Slivka AP: Angiographic assessment of pial collaterals as a prognostic indicator following intra-arterial thrombolysis for acute ischemic stroke. AJNR Am J Neuroradiol 2005;26:1789-1797.

29 Bang OY, Saver JL, Kim SJ, Kim GM, Chung CS, Ovbiagele B, Lee KH, Liebeskind DS: Collateral flow predicts response to endovascular therapy for acute ischemic stroke. Stroke 2011;42:693-699.

-30 Wechsler LR, Roberts R, Furlan AJ, et al: Factors influencing outcome and treatment effect in PROACT II. Stroke 2003;34:1224-1229.

-31 Brinjikji W, Rabinstein AA, Kallmes DF, Cloft HJ: Patient outcomes with endovascular embolectomy therapy for acute ischemic stroke: a study of the national inpatient sample: 2006-2008. Stroke 2011;42:1648-1652.

-32 Khatri P, Abruzzo T, Yeatts SD, Nichols C, Broderick JP, Tomsick TA: Good clinical outcome after ischemic stroke with successful revascularization is time-dependent. Neurology 2009;73:1066-1072.

-33 Lees KR, Bluhmki E, von Kummer R, et al: Time to treatment with intravenous alteplase and outcome in stroke: an updated pooled analysis of ECASS, ATLANTIS, NINDS, and EPITHET trials. Lancet 2010;375:1695-1703.

-34 Becktepe JS, You SJ, Berkefeld J, Neumann-Haefelin T, Singer OC: Clinical outcome after mechanical recanalization as mono- or adjunctive therapy in acute stroke: importance of time to recanalization. Cerebrovasc Dis 2011;32:211-218. 